\title{
INVERSION OF THE T WAVES IN LEAD II CAUSED BY A VARIATION IN POSITION OF THE HEART
}

\author{
BY \\ PAUL D. WHITE, FRANCIS L. CHAMBERLAIN, AND ASHTON \\ GRAYBIEL
}

\author{
From the Cardiac Laboratory and Clinics of the Massachusetts General Hospital, \\ Boston, Mass, U.S.A. \\ Received September 9, 1941
}

Only in late years has there begun to be adequate recognition of the wide variations of the normal human electrocardiogram. As has so often happened in medical science, diseased conditions and abnormal findings have attracted so much attention that a patient study of the normal subject and his physiological variations has been left far behind. This is particularly true in clinical electrocardiography where we still depend for our normal criteria on a relatively small number of records.

Leimdörfer (1935) reported the finding of a lowering, to an isoelectric or diphasic position, of the T waves of lead II in certain subjects on changing their body position from supine recumbency to standing.* This alteration of the $T$ waves he ascribed to heart disease, not otherwise apparent; for this viewpoint, however, he had no adequate evidence. That same year, two of us (P.D.W. and A.G., 1935) published a paper in which we called attention to the occasional occurrence of inversion of the $\mathrm{T}$ waves in lead II in persons without heart disease and in fact sometimes without any obvious disease at all. Several of these were young people of delicate build and subject to neurocirculatory asthenia. We stated that the reason for $T$ wave inversion in these cases was unknown but that "the importance of this finding is that it points to the need of caution in the diagnosis of serious heart disease when inversion of the $T$ wave in lead II is the only abnormal finding." In these cases the $T$ waves in lead III were always more deeply inverted than those in lead II and " remained inverted in most instances (16 out of 23 ) after the $T$ wave in lead II had ceased to be inverted."

As time went on after our own initial report we became cognizant of at least one of the factors which was responsible for the inversion of the $T$ waves

* The records taken in the standing position were made with the breath held in full expiration with the express purpose of neutralizing the effect of the change in the position of the heart; it is our experience that with ordinary breathing in the upright position the $T$ waves would often have been inverted in lead II, rather than merely isoelectric or diphasic, and that change in position of the heart with expiration alone may be insufficient to neutralize the effect of change in body position. 


\section{PAUL WHITE, F. L. CHAMBERLAIN, AND A. GRAYBIEL}

in leads II and III. We observed that the position of the body was of major importance and that a change from the upright position (seated) to recumbency (supine) often altered the $\mathrm{T}$ waves so that they became upright in lead II and less inverted, flat, or even upright in lead III. In 1938 we reported our findings in brief before the American Society for Clinical Investigation (by title and abstract) and before the New England Heart Association. We stated then that the electrocardiograms usually became normal with changes of position and after exercise. Electrocardiographic abnormalities reversible with changes in posture (sitting to supine) were often seen as transient phenomena associated with respiratory infections. The patients were mostly young adults of a nervous temperament and of asthenic habitus. Myocardial function, electrolyte balance, and heart position in relation to various body positions were studied and only the latter found to have a bearing on the electrocardiographic changes.

A variation similar to what we had encountered was reported by $\AA$ kesson (1936) and ascribed to orthostatic coronary insufficiency. He observed, among other things, that the greater the increase in pulse rate and the greater the fall in systolic blood pressure, when the subject stood erect, the greater was the decrease in the height of $\mathrm{T}$ in lead III. Akesson, however, failed to distinguish tetween the $T$ wave changes that occur immediately on assuming the upright position and those that occur as a result of the pooling of blood in the lower portions of the body. We too have observed significant changes in the $T$ waves of the electrocardiogram associated with orthostatic hypotension and tachycardia, and believe they are due to alterations in sympathetic and vagal tonus and perhaps to a decrease in coronary blood flow. However, in the observations which we report below, this mechanism did not play a significant rôle.

Sigler (1938) reported the occasional occurrence of electrocardiographic alterations with change in tcdy posiure including in a few cases inversion of the $T$ waves in lead II when the subject assumed the erect position, which he ascribed to " a change in contact of the adjacent conducting media with different portions of the heart on alteration of body posture, producing variation in conduction," and not to heart disease as Leimdörfer had done.

Two years ago an important observation came to our attention in helping to clear up the mystery of the inversion of the $T$ waves in lead II in certain normal subjects. It was already evident that posture was important but just how a change in posture acted was not wholly clear. We now found that without a change in posture the typical changes in the $T$ waves described above could sometimes be produced by deep inspiration and sometimes abolished by deep expiration. In other words, the height of the diaphragm is almost, if not quite, as important a factor as is body position, thus pointing to heart position in relation to other parts of the body as of prime significance. Rotation of the heart on its anteroposterior axis is probably a minor cause of the change, since often there is but little change in the angle of the electrical axis of the heart in the classical frontal plane of the body as measured from the QRS waves by the Einthoven formula or triangle. Rotation of the heart on its longitudinal axis is probably a more important factor in influencing the $T$ waves with change in the heart's position. Nevertheless, it should be 
recognized that the electrical axis of the $T$ waves and of the QRS complexes are distinguishable and that an alteration or shift in the QRS axis need not be associated with a comparable shift in the $T$ axis and vice versa.

Electrocardiograms illustrating the effect of posture and of respiration on the $\mathrm{T}$ waves in leads II and III were published by two of us (P.D.W. and A.G.) in our new book on electrocardiography (1941). Records of one case (Fig. 15 and 16 of the book) are reproduced herewith as Fig. 1. Other typical illus-

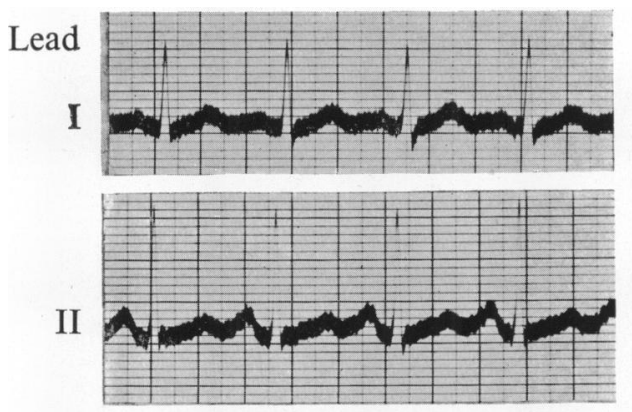

III

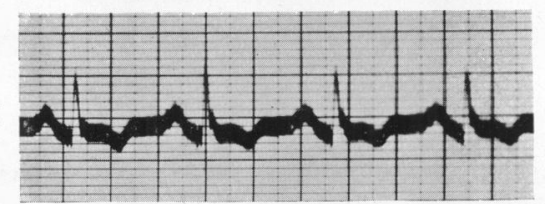

IV

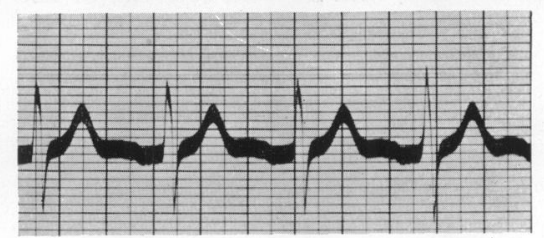

A
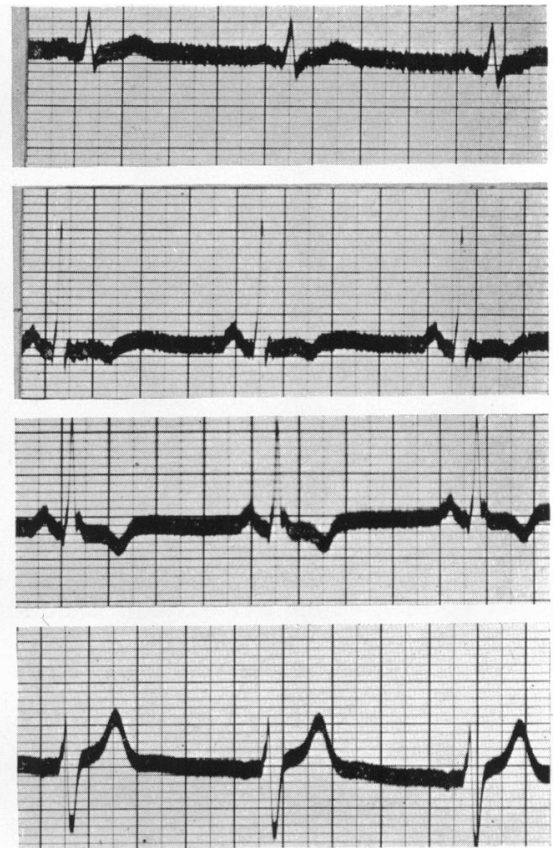

B

FIG. 1.-Electrocardiographic changes with respiration in a healthy young man of stocky build. (A) During quiet respiration. (B) In full inspiration.

Note that here there is a distinct shift in the angle of the electrical axis of the QRS waves to the right as well as the inversion of the T waves in lead II upon lowering the diaphragm and making the heart much more vertical in its position. There may or may not, however, be much of any change in the QRS waves with change in the T waves.

trations are shown in Fig. 2, 3, 4, and 5 of the present paper. It should be noted that changes in the S-T junctions and segments and in the $T$ waves in leads I and IV are relatively slight and unimportant in comparison with the changes in the late phases of the T waves in leads II and III.

Recently Scherf and Weissberg (1941) have reviewed the subject and have arrived at the same conclusions as ourselves; they pointed out that the $T$ waves in lead II may be simply lowered rather than inverted, and emphasized particularly the inversion of $\mathrm{T}$ in lead III and its change in 35 normal adults. They concluded that their "observations speak against the assumption that cardiac damage or anoxia of the heart muscle can be the factor responsible for the electrocardiographic alterations. They confirm the conception that the 
Lead
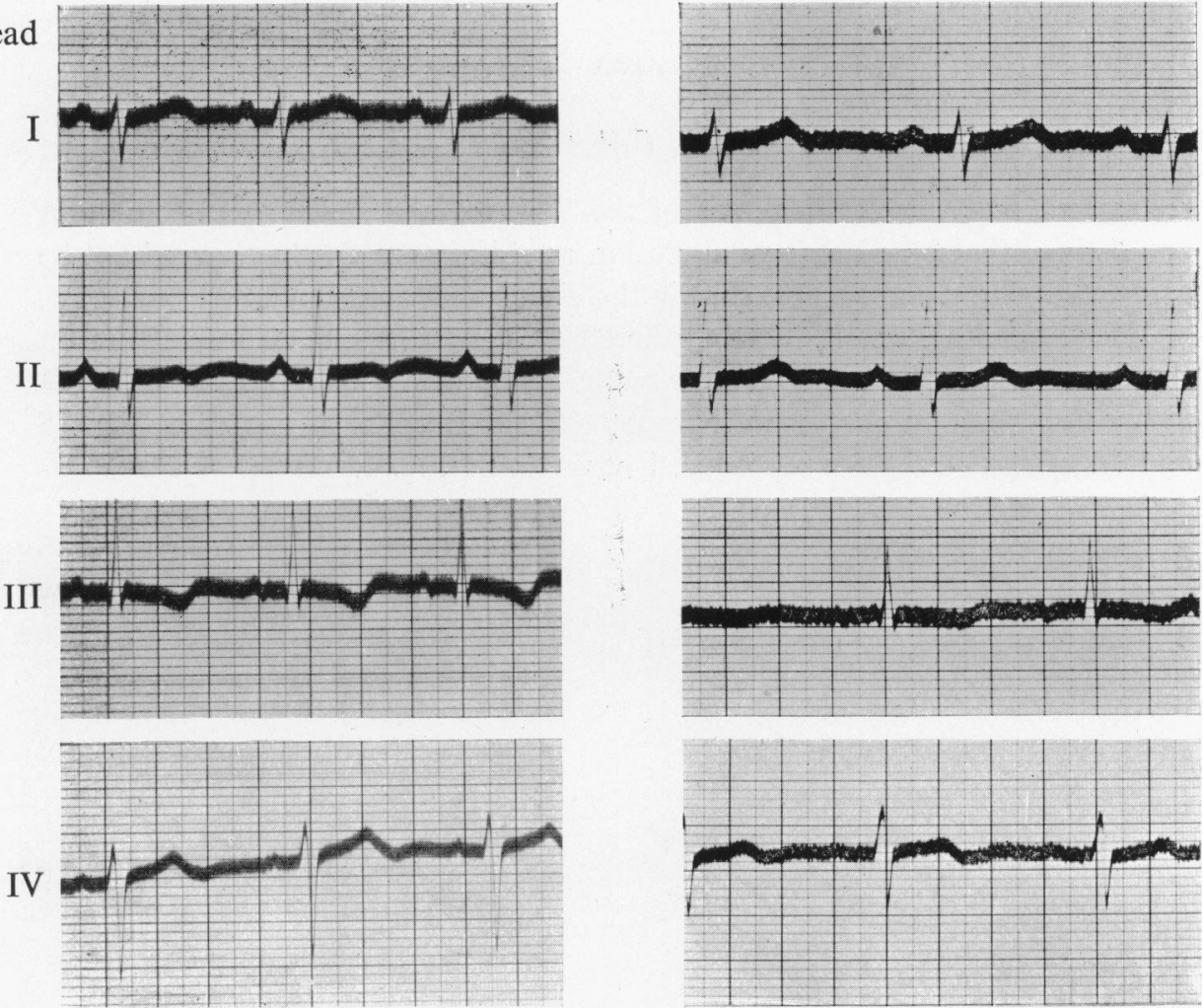

A

B

Fig. 2.-Electrocardiographic changes upon change in position from sitting to supine in the case of a young girl, 15 years old and of average build, with no clear evidence of heart disease but of nervous type.

(A) Sitting.

(B) Recumbent.

Lead
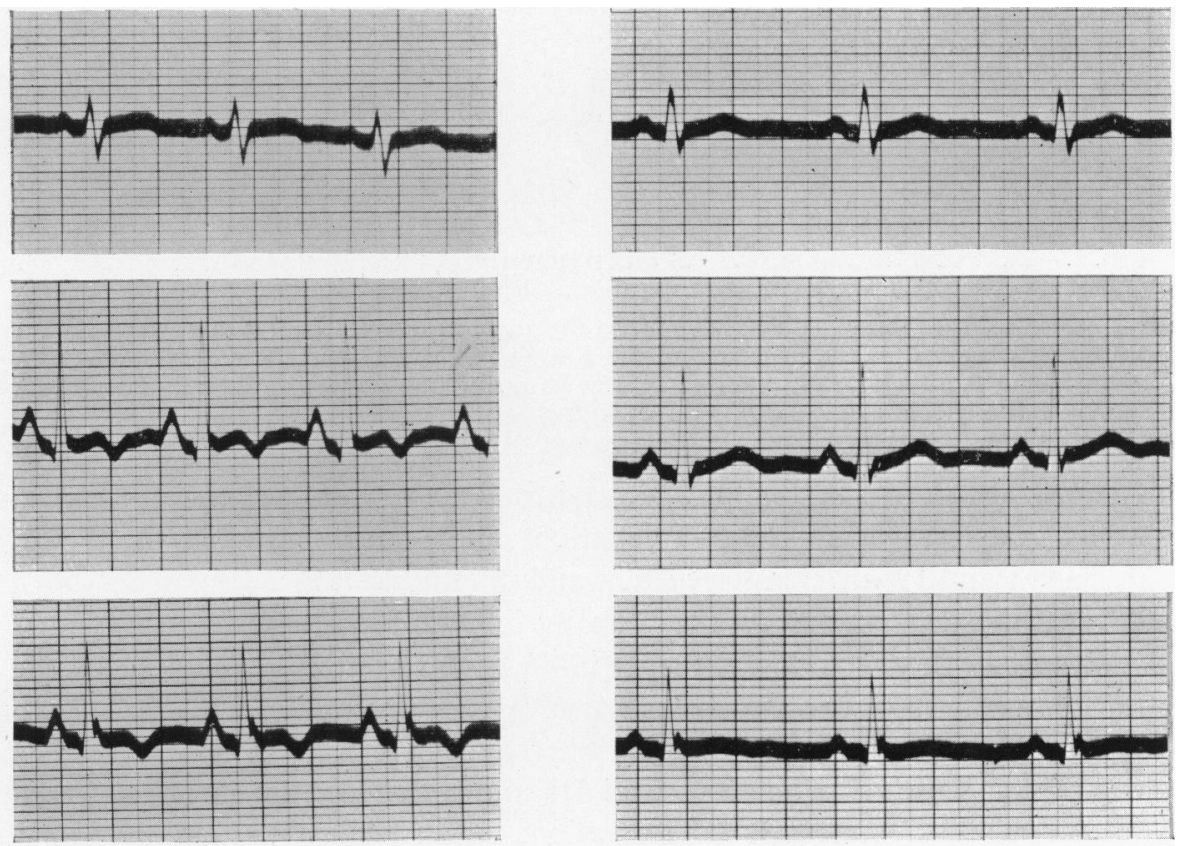

A

B

FIG. 3.-Electrocardiographic changes upon change in position from sitting to supine in the case of a young girl of 18 , tall and thin, without evidence of heart disease.
(A) Sitting.
(B) Recumbent. 


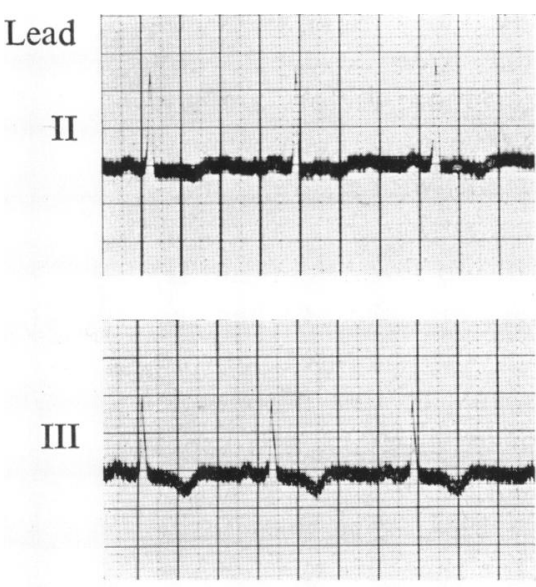

A
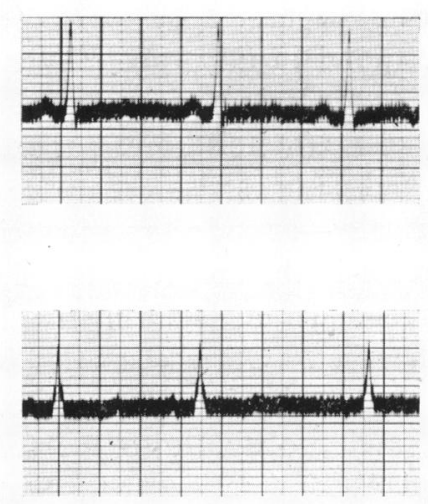

B
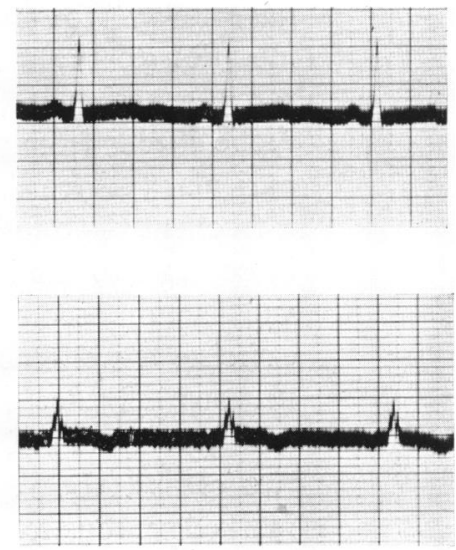

C

Fig. 4.-Electrocardiographic changes in leads II and III upon change of position from sitting to supine and upon full expiration, in the case of a boy of 15 , tall and thin, with a normal heart, vertical in position.

(A) Sitting, during quiet respiration. (B) Recumbent. (C) Sitting, at end of full expiration.

inversion of the $\mathrm{T}$ wave is due to a change in posture and therefore a change in contact between the heart and the neighbouring tissues."

\section{DisCUSSION}

Lowering, flattening, notching, and inversion of the $\mathrm{T}$ waves in lead II are the result of a variety of causes, mostly diseases involving the heart itself. The majority of these are well recognized, ranging from the common changes due to coronary heart disease to the rare findings in myxœdema. Pericarditis, acute and chronic, is an occasional but important cause, acting doubtless through the subjacent myocardial involvement. Myocarditis of rheumatic or other rarer nature can cause such $\mathrm{T}$ wave changes, as can also the toxic effects associated with severe infections, renal toxemias and drug action, particularly the well-known intoxication by digitalis. Pronounced enlargement and strain of the right ventricle, acute or chronic, as in the cor pulmonale or in marked mitral stenosis, may cause diphasic flattening or inversion of the $\mathrm{T}$ waves in lead II as well as of those in lead III.

The abnormal causes of the changes in T in lead II listed in the last paragraph have been recognized for the most part for years, but interestingly enough the normal or physiological factors responsible for very similar changes have only begun to receive recognition long overdue. Even as recently as 1935 Leimdörfer, as stated above, assumed that such changes without evidence of heart disease or intoxication must of necessity mean that heart disease, particularly coronary insufficiency, is none the less present. Now we are learning better. 
There are several physiological causes for changes of $\mathrm{T}$ in lead II, but these have not yet been clearly separated by the few writers who have paid any attention to the subject, and in all probability two or more factors are not rarely superimposed. Autonomic nervous influences (preponderantly sympathetic effects) from any cause (exercise, fever, or excitement) with resulting sinoauricular tachycardia, and perhaps also even paroxymal auricular tachycardia, is frequently-in fact usually-attended by a lowering of the $\mathrm{T}$ waves in lead II, in contrast to the increase in the height of the $T$ waves that occurs when the vagal action becomes preponderant as during the slowing of the heart following exercise, a well-known effect. Other examples of causes of this autonomic nervous influence are thyrotoxicosis, which we recognized in 1935, and vagal paralysis by atropine, and probably also the toxic effect of tobacco which two of us (P.D.W. and A.G.) described with Starr in 1938. Fright, with its marked nervous reflex effect, is probably another cause for change in the $T$ waves of this type, as will be reported later by ourselves. Thus the presence of considerable tachycardia should make one suspicious of the possible or probable action of this factor when the $T$ waves are low or even inverted in lead II. The sitting and particularly the standing position favours the development of a relative tachycardia compared with the heart rate in recumbency, and so this naturally may be one reason, but we believe not the major reason, for the change of the $\mathrm{T}$ waves in lead II with change in posture. Barker and his associates (1939) have also observed significant lowering of the $T$ waves of the electrocardiogram resulting from overventilation with alkalosis, which, as a symptom of anxiety or hysteria, is met with occasionally. (See addendum on p. 240.)

It is, however, the change in position of the heart in the thorax, which occurs particularly in the case of the vertical or drop heart with the subject in the standing or sitting position, that concerns us particularly here. The $T$ waves in lead II are often simply lower than usual, but sometimes they are flat or diphasic, and occasionally they are actually inverted with a simulation of a diseased state, particularly coronary heart disease. The lateness of the inversion of the $T$ waves adds considerably to this simulation of a disease pattern. There is, however, one $T$ wave pattern, namely, that pictured in Fig. 5, that is almost if not wholly pathognomonic of this positional effect, duplicated rarely if at all by pericarditis or coronary heart disease: that is a late slight notching of the $\mathrm{T}$ wave, the notch sometimes scarcely depressing the baseline below its zero position. This finding has been an important clue for us in a number of cases.

It behoves us, therefore, when we encounter flattening, notching, or inversion of the $\mathrm{T}$ waves in lead II in apparently healthy persons, especially in those who are young and of a slim build with vertical hearts (the very type who are prone to develop neurocirculatory asthenia and anxiety with overventilation) to take electrocardiograms with the subject in the recumbent (supine) posture as well as sitting, and to be sure that the subject is composed and not excited or fearful. It is also always important to record the position of the body when electrocardiograms are taken for the sake of present and future reference, because 
Lead

I

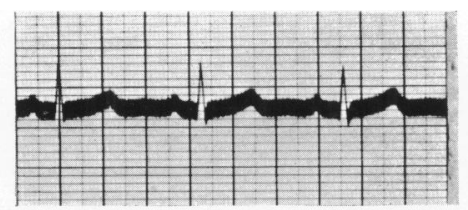

II

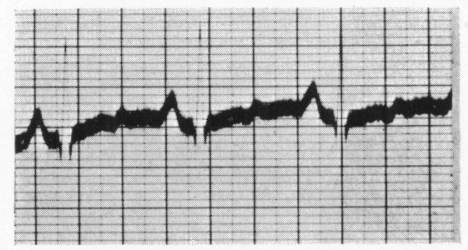

III

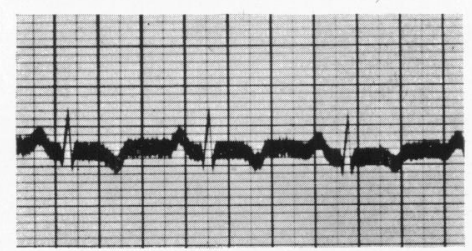

IV

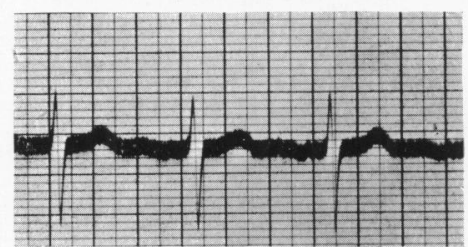

A
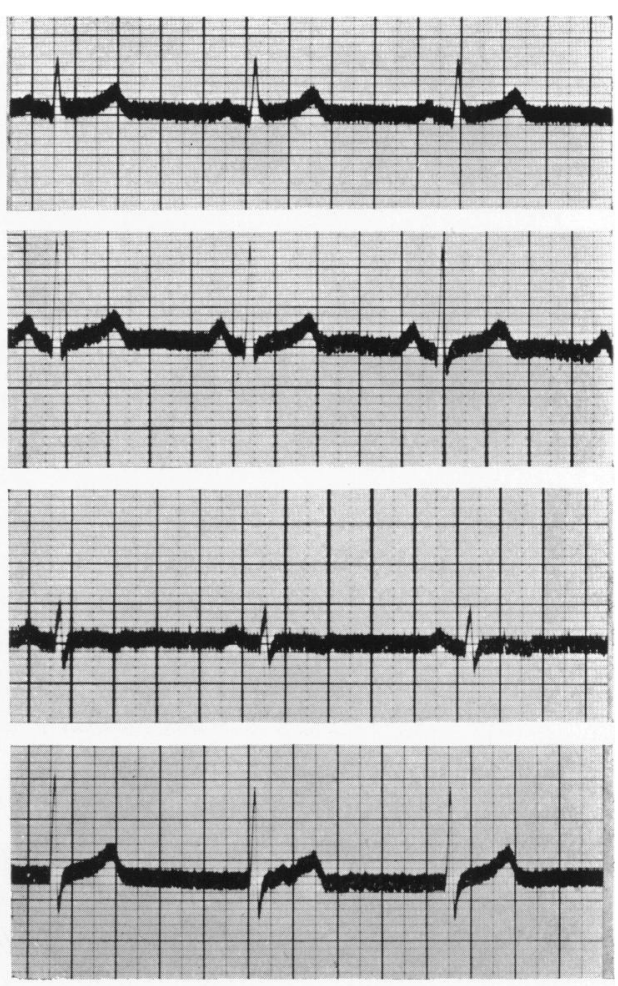

B

FIG. 5.-Electrocardiographic changes upon change in position from sitting to supine in the case of a woman 45 years old of average build without evidence of heart disease.
(A) Sitting.
(B) Recumbent.

of the possible errors in interpretation that may easily arise especially when comparing a series of electrocardiograms. A simple way of recording the position accurately is to draw a straight line which shows whether the thorax is horizontal, vertical, or at a midway angle, thus - 1 .

Also we should recognize that in these cases posture alone is not the whole answer. It is the position of the heart that counts, and, as has been shown by Scherf and Weissberg and by ourselves, the height of the diaphragm is often very significant, its elevation at full expiration tending to "correct" the $T$ waves, though often not so completely as does recumbency.

Follow-up studies during the past few years, in some cases now extending back nearly ten years, have supported our conviction that the changes we have described in the $\mathrm{T}$ waves of lead II are not the result of disease. Our cases have not developed any evidence of abnormality of the heart, and a few who were frail and nervous (with tachycardia) when first seen have grown more robust and composed with improved health in the course of years, and have manifested a "correction" of the $T$ wave variations even in the sitting position; it is probable that a raising of the height of the diaphragm and a decrease in sympathetic overstimulation and hyperventilation account for this 
" correction" which was cited but not explained by two of us (P.D.W. and A.G.) in 1935. Also it is, of course, very significant that our most striking cases have been asthenic young people.

Finally, this " normal inversion " of the T waves in lead II is quite a common finding. We now recognize several new cases every month in our routine electrocardiographic interpretations and a number of these have been referred to us with the diagnosis of heart disease, often obscure, on the basis of these electrocardiographic findings alone, or with the addition of neurocirculatory asthenia or a physiological heart murmur. Orthostatic hypotension, with tachycardia, in a rare case may be attended by significant $\mathrm{T}$ wave inversion, as we have already noted above; whether or not in such cases a decrease in coronary blood flow enters in as a factor we cannot say. It should be realized, of course, that there may be a combination of physiological and pathological factors in the alteration of the $\mathrm{T}$ waves in lead II in the same case; a tall lean person may have pericarditis or coronary heart disease or nervous overventilating.

\section{SUMMARY}

Inversion of the $\mathrm{T}$ waves in lead II of the electrocardiogram, although most commonly the result of heart disease or toxic states, may be a normal physiological variation in occasional persons, particularly those of asthenic habitus with vertical hearts and prone to neurocirculatory asthenia.

The position of the heart is the most important factor in producing this $T$ wave inversion which is found in the sitting or standing position but is corrected by recumbency or by elevating the diaphragm as at full expiration. Autonomic nervous influences comprise another factor, although less striking as a rule, the low or inverted $T$ waves then being attended by tachycardia; any cause of such stimulation, for example, excitement can then be responsible. Fear and anxiety may act through the production of overventilation with resultant alkalosis. Both heart position and nervous influences may be active in the same case.

The relatively common occurrence normally of inversion of the $T$ waves in lead II makes it imperative to recognize its existence in order to avoid erroneous diagnoses of heart disease.

Addendum.-Finally, Mainzer and Krause (1940), described the influence of fear on the electrocardiogram. Immediately before the induction of general anaesthesia, electrocardiographic changes were noted in about two-fifths of 53 cases; these changes consisted chiefly of S-T depression and lowering or inversion of the $T$ waves similar to that appearing in coronary insufficiency and ascribed by the authors to neurogenic influences reducing the coronary flow.

\section{REFERENCES}

Akesson, S. (1936). Upsala läkaref. forh., 42, 263.

Barker, P. S., Shrader, E. L., and Ronzoni, E. (1939). Amer. Heart J., 17, 169.

Graybiel, A., and White, P. D. (1935). Ibid., 10, 345.

Graybiel, A., Starr, Robert S., and White, P. D. (1938). Ibid., 15, 89.

Graybiel, A., and White, P. D. (1941). Electrocardiography in Practice, Philadelphia.

Leimdörfer, A. (1935). Med. Klin., 31, 1536.

Mainzer, F. and Krause, M. (1940). Brit. Heart J., 2, 221.

Scherf, D., and Weissberg, J. (1941). Amer. J. med. Sci., 201, 693.

Sigler, L. H. (1938), Amer. Heart J., 15, 146.

White, P. D., and Chamberlain, F. L. (1938). J. Clin. Invest., 17, 510.

White. P. D., and Chamberlain, F. L. (1938). New England Heart Association Abstracts. 